\title{
Anemia in Cardio-Renal Syndrome: clinical impact and pathophysiologic mechanisms
}

\author{
Alberto Palazzuoli • Giovanni Antonelli • \\ Ranuccio Nuti
}

Published online: 19 February 2011

(C) Springer Science+Business Media, LLC 2011

\begin{abstract}
Anemia is a disease that is often associated with heart failure (HF) and renal insufficiency (RI). This unfavorable triad of conditions has been called CardioRenal-Anemia Syndrome (CRS). The association of HF, $\mathrm{RI}$, and anemia is poorly reported in multicenter clinical trials, so the pathophysiologic mechanisms and treatment options need to be better defined. When CRS patients develop anemia, a "perfect storm" often occurs: HF and RI cause anemia which will worsen the first two conditions. Anemia appears to be the result of complex interactions between cardiac performance, bone marrow homeostasis, renal dysfunction, and various drug side effects. However, neurohormonal and inflammatory activities play a key role in the beginning and progression of the disease. As a consequence, endogenous erythropoietin activity dysfunction with inadequate production and tissue resistance occurs. Despite the advances of therapy in the neurohormonal activation blockade, mortality and hospitalization in HF still remain unacceptably high, suggesting that specific comorbidity treatments could have a significant positive prognostic impact. Anemia should be recognized as one of the novel targets in HF treatment.
\end{abstract}

Keywords Anemia - Cardio-Renal Syndrome Heart failure

A. Palazzuoli $(\bowtie) \cdot$ G. Antonelli · R. Nuti Department of Internal Medicine and Metabolic Diseases, Cardiology Section, S Maria alle Scotte Hospital University of Siena, Le Scotte Hospital, Viale Bracci, 53100 Siena, Italy e-mail: palazzuoli2@unisi.it

\section{Introduction}

Anemia as well as renal insufficiency (RI) are two conditions that tend to be excluded from large clinical trials. The prevalence of anemia in multicenter studies varies from 5 to $55 \%$. This has to do with the clinical selection criteria as well as with different cutoff points [1]. However, anemia has been recognized as an important comorbidity in heart failure (HF) patients, and it appears to be related to the worse outcome in patients with both RI and HF. Cardio-Renal Syndrome (CRS) is often associated with anemia [2]. This unfavorable triad of conditions has been called Cardio-Renal-Anemia Syndrome (CRAS). Multiple observational studies have clearly demonstrated an independent association between lower hemoglobin values and an adverse clinical outcome. Current HF guidelines do not provide specific recommendations for the evaluation and treatment of anemia [3]. Since the mortality in HF remains high despite the therapeutic advances in the neurohormonal activation blockade, anemia could represent one of the novel therapeutic targets in HF.

\section{Clinical impact of anemia in $\mathrm{HF}$}

Anemia is often associated with such unfavorable clinical conditions as older age, diabetes, RI, and a high incidence of atherosclerotic burden as cerebral and coronary disease [4]. The ARIC study demonstrated that anemia in primary prevention is related to a higher incidence of cardiovascular diseases [5]. HF patients with anemia are more likely to have a worse quality of life as they have a lower exercise capacity, a higher NYHA classification, lower blood pressure, hyponatremia, and the need for higher 
diuretic dosages. The Optimize-HF registry containing information about more than 48,000 patients reported that anemia is associated with all-cause mortality and morbidity, and an increased relative risk of around 30\% [6]. Similarly, Kosiborod et al. [7] demonstrated a progressively worsening outcome for hematocrit values below $32 \%$, and patients with $27 \%$ demonstrated a similar prognostic impact as patients with an ejection fraction lower than $25 \%$. Two recent meta-analysis evaluating hemoglobin levels as a continuous variable confirmed this trend. In both studies, anemia was frequently observed and was found in over one-third of HF patients: the unadjusted mortality risk was OR 1.96, and after analyzing multivariate assessments including sociodemographic variables, medical history, drug use, and laboratory data as parameters, anemia remained an independent risk factor for mortality $[8,9]$. These data could be extended to patients with systolic or diastolic HF, in fact data from the CHARM study revealed similar risk factors for cardiovascular death and hospitalization in both groups. Therefore, anemia was similarly present in patients with preserved or depressed ejection fraction and in most cases associated with RI [10]. The data reported referred to a basal $\mathrm{Hb}$ level; however, a $\mathrm{Hb}$ decrease of more than $1 \mathrm{~g} / \mathrm{dl}$ during a follow-up period has been independently associated with subsequent mortality and morbidity. $\mathrm{A} \mathrm{Hb}$ decrease appears also to be related to several other variables including BNP, CRP, albumin levels, and the glomerular filtration rate. Similar data are reported for less serious degrees: ambulatory patients with anemia displayed a mortality risk in 5 years of $18 \%$ with respect to non-anemic patients. Even in this population, anemia is associated with higher BNP levels, poor diastolic function, and increased pulmonary artery pressure [11].

Anemia and RI are often associated, but the only study that analyzed the combination of the two diseases is the ANCHOR Trial, evaluating 58,000 patients with HF, RI, and anemia during a 5-year follow-up. In this study, the independent prognostic impact of both $\mathrm{Hb}$ and GFR with a progressive crude mortality risk below $11 \mathrm{~g} / \mathrm{dl}$ and $44 \mathrm{ml} /$ min, respectively, was clearly demonstrated [12].

The same results regarding HF patients could include subjects suffering from coronary artery disease: in this setting, anemia is associated with greater coronary disease extension and higher adverse events in stable angina as well as in acute coronary syndromes. Moreover, anemic coronary patients demonstrated higher mortality and hospitalization risks [13]. In patients submitted to percutaneous revascularization, anemia is associated with increased troponin and myoglobin periprocedural levels which is likely due to the reduced levels of plasma $\mathrm{O}_{2}$ tension [14].

\section{Compensatory mechanisms and consequences of anemia}

The mechanisms behind the increased mortality risks observed in anemic CHF patients are not completely understood. Anemia may lead to an increased cardiac work, resulting from increased heart rate, stroke volume, and workload [15]. Renal failure might be implicated as well in the pathophysiology as a common comorbidity in CHF. The most important compensatory mechanisms caused by the reduced oxygen tension are nonhemodynamic and hemodynamic responses, which are shown in Table 1.

Non-hemodynamic responses to anemia include an increase in erythropoiesis to enhance the oxygen-carrying capacity, as well as an increase in RBC 2,3-diphosphoglycerate that shifts the hemoglobin-oxygen dissociation curve to the right to improve the oxygen delivery to the peripheral tissues. The $\mathrm{Hb}$-oxygen dissociation curve is influenced by several factors: tissue acidosis and an elevated diphosphoglycerate concentration which shift the curve to the right, a decreasing $\mathrm{Hb}$ affinity for oxygen with consequent improvement of the oxygen delivery $[2,16]$. Since the erythropoiesis is defective in HF, the hemodynamic responses may predominate. The hemodynamic response is complex, depending on a delicate balance between the vasodilation status and the neurohormonal activation. The initial hemodynamic response to anemia is a decrease of the vascular resistance. This decrease of the vascular resistance causes a reduction of the systemic blood pressure. Both mechanisms are able to trigger a baroceptor response throughout neurohormonal activation. The increase of the renin-angiotensin-aldosterone levels and the sympathetic activity will lead to a peripheral vasoconstriction, a reduction in renal blood flow and glomerular filtration rate, as well as salt and water retention with a subsequent expansion of the extracellular plasma volume. The extracellular plasma expansion associated with vasodilation causes an early cardiac output increase. However, after a short-term response, systemic and venous congestion will set in, worsening the HF status [17].

Table 1 Hemodynamic and non-hemodynamic compensatory mechanisms of Anemia in Heart Failure

\begin{tabular}{ll}
\hline Non-hemodynamic & Hemodynamic \\
\hline Increase erythropoiesis & \\
Affinity change $\mathrm{Hb} / \mathrm{O}_{2}$ & Increase LV work load and output \\
& Reduced vascular resistance \\
& Increased RAA activity \\
& Reduced GFR and renal blood flow \\
& Salt and water retention \\
\hline
\end{tabular}




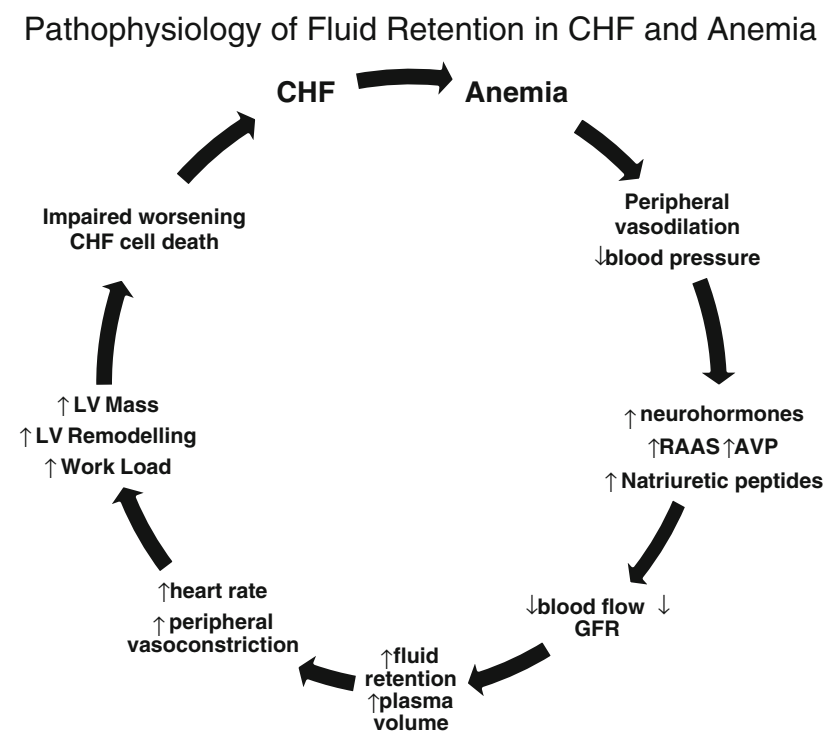

Fig. 1 Physio-pathologic cascade during heart failure and anemia: neurohormonal and inflammatory activation worsen renal blood flow and increase extracellular fluid volume balance with further impairment of cardiac morphology and function

The cathecolamine and RAA activity associated with sodium reuptake and the increase in water retention will cause deleterious direct effects on the cardiac muscle: the higher cardiac workload increases the left ventricular mass and remodels it with a higher myocardial fibrosis deposition, myocardial disarray, and subsequent risks of arrhythmia [18]. All these cardiac modifications could result in worsening heart failure and have a potential adverse outcome. Hemodynamic and neurohormonal responses to anemia are very similar to the pathophysiologic mechanisms seen in HF and RI. Both diseases could create a vicious circle where HF causes RI and both cause anemia which will worsen both HF and RI (Fig. 1).

\section{Causes of anemia}

Although the causal relationship between HF and anemia remains poorly defined, there are multiple potential mechanisms throughout which the HF syndrome could contribute to the development of anemia. Several potential mechanisms may be involved, including hematinic abnormalities linked to reduced bone perfusion, hemodilution, the inflammatory status activity, malnutrition, malabsorption, renal insufficiency with renal blood flow modifications, as well as side effects of drugs currently used in the treatment of HF $[1,17]$.

Hematinic abnormalities

Serum vitamin B12 and folic acid levels are low in just a minority of anemic patients with HF. Similarly iron deficiency, although insufficiently reported in the literature, could represent a contributing factor. Gastrointestinal bleeding from aspirin, warfarin-like agents, malignant tumors, polyps or esophagitis, or reduced iron absorption resulting from gastrointestinal diseases could potentially contribute to the anemia.

\section{Hemodilution}

An expanded plasma volume is very common in HF and RI. Hemodilution can even occur in euvolemic patients inducing a false anemic status [18]. However, a recent study demonstrated that a red cell deficit was present in most of CHF patients with anemia. All systolic CHF patients and $71 \%$ of the diastolic CHF patients presented an expanded plasma volume. This study suggests that there is a true red cell deficit in the majority of anemic CHF patients [19].

\section{Inflammatory activity}

Proinflammatory cytokines such as tumor necrosis factor (TNF)-alpha and interleukin-6 have direct effects on the bone marrow, and are implicated in the mechanism of chronic anemia. High levels of circulating proinflammatory cytokines are known to be associated with greater disease severity and worsened clinical outcomes. Elevated interleukins levels lead to reduced EPO production in the kidneys, reduced EPO activity in the bone marrow, bone tissue resistance to EPO (with a decrease in erythroid progenitor cell production), and an increase in the hepcidin production in the liver [20]. An increased production of hepcidin reduces ferroportin in the gut, reducing iron absorption as well as reducing iron release from iron stores in macrophages and liver cells. Thus, the inflammatory pathway appears to be one of the key components for the development of anemia as a chronic disease in $\operatorname{HF}[21,22]$.

Malnutrition and malabsorption

Anemia is often associated with a poor nutritional status and low albumin levels. Reduced absorption due to edema of the mucosae at the gastrointestinal level caused by rightsided heart failure could contribute to worsening anemia. Therefore, malnutrition occurs frequently with a cachectic status and reduced hunger stimulus.

Renal insufficiency

Renal insufficiency is common in CHF, it is associated with an increased risk of progression of renal disease, sudden death, and cardiovascular mortality. The renal damage is probably mainly due to reduced renal blood flow 
as well as intrarenal flow alteration causing hypoxic renal damage. The increased peritubular capillary oncotic pressure is associated with reduced peritubular hydrostatic capillary pressure, which increases sodium resorption [23]. Therefore, parenchymal alterations are often associated with HF, including tubular loss, tubulointerstitial fibrosis, and vascular obliteration. In patients with $\mathrm{HF}$ and RI, progressive renal dysfunction leads to a decrease in circulating levels of erythropoietin, with a subsequent decrease in bone marrow erythrocyte production and $\mathrm{Hb}$ levels [8].

Drugs side effects

Angiotensin is a potent stimulus for the erythropoietin production. The use of ACE inhibitors could cause a decrease in circulating EPO levels with a modest $\mathrm{Hb}$ reduction. Similarly, the use of Angiotensin II receptor blockers is associated with a higher incidence of anemia [24]. Beta-blockers are also associated with a higher incidence of anemia. Therefore, the drug blockade leads to a resistance to EPO and a reduced genetic EPO expression [25].

In particular, a specific blockade of beta- 2 adrenergic receptors could potentially abolish both the sympathetically mediated increase in erythropoietin production and the erythroid progenitor cell proliferation [26].

\section{EPO and iron metabolism in CRS anemic patients}

Several mechanisms are involved in the development of anemia, even if the causal relation linking HF to anemia remains poorly defined. Many of the above-cited mechanisms are simultaneously activated, and the $\mathrm{Hb}$ reduction in $\mathrm{HF}$ is the result of complex interactions between renal insufficiency, inflammatory activation, bone marrow dysfunction, and increased neurohormonal activity. All these phenomena have a common target: erythropoietin (EPO) production, resistance, and downregulation [27]. EPO is a glycoprotein growth factor produced primarily by the kidneys; it is the key component of the homeostatic system for the regulation of the red blood cell mass and the tissue oxygen delivery. It stimulates the erythroid cell proliferation, maturation, and differentiation. During CRS, a blunted EPO production associated with insufficient EPO production occurs [28]. In a pilot study by Opasich et al. [20] evaluating patients with $\mathrm{HF}$ and anemia, the authors found that one-third of the patients had renal dysfunction with consequent reduced EPO production. On the other hand, other studies showed that patients with increased endogenous EPO levels had a worse outcome. However, in the same sample, there was a lack of correlation between HB and EPO levels with the serum levels being disproportionately low for the degree
Table 2 Iron and erythropoietin metabolism alterations associated with renal dysfunction and inflammatory activation

EPO and iron metabolism

Blunted EPO production

Reduced EPO genetic expression

EPO receptors down-regulation

Alterated EPO signal transduction

Reduced iron transport and absorption

Increase Hepcidin expression mediated by inflammation

Interference between Hepcidin and ferroprotein

of anemia $[29,30]$. These data confirm that in HF as well as in $\mathrm{RI}$, there is a relative EPO deficency and a bone marrow resistance to endogenous EPO. Altered EPO metabolism causes a reduced production and activity as well as other dysfunctions: impaired erythroid progenitor cell proliferation, reduced gene expression of EPO, altered EPO signal transduction, and a limited availability of iron for the erythropoiesis. Another cause of the blunted EPO response is the decreased EPO receptor number due to their internalization. All these phenomena appear to be mediated mainly by two conditions. (1) Kidney dysfunction: EPO is mostly produced by the kidneys, and hypoxia is the fundamental physiologic stimulus to the EPO renal production through an exponential increase in the number of EPO producing cells. (2) Inflammatory cytokines have a direct cytotoxic effect on erythroid progenitor cells and inhibit their growth. Therefore, interleukins and TNF-alfa have been shown to disrupt multiple aspects of erythropoiesis, including reduction in the renal EPO secretion, suppression of the EPO activity on red blood cell precursors in the bone marrow level, and the reduction in the bioavailability of iron storage [31] (Table 2).

The other alteration involves the iron metabolism: iron is essential for the $\mathrm{Hb}$ synthesis, and it's homeostasis involves iron absorption from the diet, iron moving from the liver, and iron recycled from older red blood cells [32]. Hepcidin is a protein produced by an inflammatory status, and it regulates negatively both iron absorption from the gastrointestinal level and iron transport by negative interaction with macrophages. Hepcidin reduces iron gut absorption, thus reducing its bioavailability for $\mathrm{Hb}$ production. Iron is recycled from senescent blood cells and released from hemoglobin after macrophage phagocytosis [33]. From the macrophages, iron is transported into the plasma by transferrin that donates iron to cells through transferring receptors.

\section{Conclusions}

Anemia is a common disease in CRS, and it is an independent prognostic risk factor. $\mathrm{Hb}$ reduction may represent 
the final phenomena of several problems ranging from neurohormonal and inflammatory activation to renal blood flow and the dysregulation of kidney structure. When CRS patients develop anemia, a perfect pathophysiologic storm occurs: HF and RI cause anemia which worsens the first two conditions. Altered EPO metabolism is a key component in this complex mechanism, and it appears mediated by the inflammatory activity. Despite the neurohormonal blockade and hemodynamic improvement by current therapy, the neutral or negative results in clinical trials during the last 10 years suggest that we have reached the ceiling of benefits in HF treatment. A "holistic" view looking for comorbidities (i.e. RI and anemia) could lead to a better classification and treatment in patients with Cardio-RenalAnemia Syndrome.

\section{References}

1. Felker GM, Adams KF, Gattis WA et al (2004) Anemia as a risk factor and therapeutic target in heart failure. J Am Coll Cardiol 44:959-966

2. Kazory A, Ross EA (2009) Anemia: the point of convergence or divergence for kidney disease and heart failure? J Am Coll Cardiol 53:639-647

3. Hunt SH, Abraham WT, Chin MH et al (2009) Focused update incorporated: into the ACC/AHA 2005 guidelines for the diagnosis and management of heart failure in adults a report of the American college of cardiology foundation/American heart association task force on practice guidelines. Circulation 119:e391-e479

4. Ezekowitz JA, McAlister FA, Armstrong PW (2003) Anemia is common in heart failure and is associated with poor outcome. Circulation 107:223-225

5. Sarnak MJ, Tighiouart H, Manjunath G et al (2002) Anemia as a risk factor for cardiovascular disease in the atherosclerosis risk in communities (ARIC) study. J Am Coll Cardiol 40:27-33

6. Young JB, Abraham WT, Albert NM et al (2008) Relation of low hemoglobin and anemia to morbidty and mortality in patients hospitalized with heart failure (insight from the OPTIMIZE-HF registry). Am J Cardiol 101:223-230

7. Kosiborod M, Smith GI, Radford MJ et al (2003) The prognostic impact of anemia in patients with heart failure. Am J Med 114: 112-119

8. Tang YD, Katz SD (2006) Anemia in chronic heart failure. Prevalence etiology clinical correlates and treatment options. Circulation 113:2454-2461

9. Groenveld HF, Januzzi JL, Damman K et al (2008) Anemia and mortality in heart failure patients. A systematic review and metaanalysis. J Am Coll Cardiol 52:818-827

10. O'Meara E, Clayton T, McEntegart MB et al (2006) Clinical correlates anc consequences of anemia in a broad spectrum of patients with heart failure. Results of the candesartan in heart failure: assessment of reduction in mortality and morbidity CHARM program. Circulation 113:986-994

11. Tang WH, Tong W, Jain A et al (2008) Evaluation and long term prognosis of new-onset transient and persistent anemia in ambulatory patients with chronic heart failure. J Am Coll Cardiol 51:569-576

12. Go AS, Yang J, Ackerson LM et al (2006) Hemoglobin level chronic kidney disease and the risks of death and hospitalization in adults with chronic heart failure. The anemia in chronic heart failure:outcomes and resource utilization (ANCHOR) study. Circulation 113:2713-2723

13. Lee PC, Kini AS, Ahsan C et al (2004) Anemia is an independent predictor of mortality after percutaneous coronary intervention. J Am Coll Cardiol 44:541-546

14. Husemann W, Fobker M, Pohlen M et al (2007) Impact of haemoglobin concentration and chronic kidney disease in patients with coronary heart disease undergoing percutaneous coronary interventions. Nephrol Dial Transpl 22:2563-2570

15. Weiskopf RB, Viele MK, Feiner J et al (1998) Human cardiovascular and metabolic response to acute severe isovolumic anemia. JAMA 279:217-221

16. Oski FA, Marshall BE, Cohen PJ et al (1971) The role of leftshifted or right-shifted oxygen haemoglobin equilibrium curve. Ann Intern Med 75:14-18

17. Anand IS (2008) Heart failure and anemia: mechanisms and pathophysiology. Heart Fail Rev 13:379-386

18. Berry C, Norrie J, Hogg K et al (2006) The prevalence, nature and importance of hematologic abnormalities in heart failure. Am Heart J 151:1313-1321

19. Witte KK, Desilva R, Chattopadhyay S et al (2004) Are hematinic deficiencies the cause of anemia in chronic heart failure? Am Heart J 147:924-930

20. Opasich C, Cazzola M, Scelsi L et al (2005) Blunted erythropoietin production and defective iron supply for erythropoiesis as a major causes of anemia in patients with chronic heart failure. Eur Heart J 26:2232-2237

21. Deswal A, Petersen NJ, Feldman AM et al (2001) Cytokines and cytokines receptors in advanced heart failure: an analysis of the cytokines database from the Vesnarinone trial (VEST). Circulation 103:2055-2059

22. MacDougall IC, Cooper AC (2002) Erythropoietin resistance: the role of inflammation and pro-inflammatory cytokines. Nephrol Dial Transplant 17(suppl 11):39-43

23. Schrier PW (2006) Role of diminished renal function in cardiovascular mortality: marker or pathogenic factor? J Am Coll Cardiol 47:1-8

24. Anand IS, Kuskowski MA, Rector TS et al (2005) Anemia and change in hemoglobin over time related to mortality and morbidity in patients with chronic heart failure results from Val-Heft. Circulation 112:1121-1127

25. Komajda M, Anker SD, Charlesworth A et al (2006) The impact of new onset anaemia on morbidity and mortality in chronic heart failure: results from COMET. Eur Heart J 27:1440-1446

26. Mladenovic J, Adamson JW (1984) Adrenergic modulation of erythropiesis: in vitro studies of clony forming cells in normal and policitaemic man. Br J Hematol 56:323-332

27. Silverberg DS, Wexler D, Iaina A (2002) The importance of anemia and its correction in the management of severe congestive heart failure. Eur J Heart Fail 4:681-686

28. Jelkmann W (2004) Molecular biology of erythropoietin. Intern Med 43:649-659

29. Van der Meer P, Voors AA, Lipsic E et al (2004) Prognostic value of plasma erythropoietin on mortality in patients with chronic heart failure. J Am Coll Cardiol 44:63-67

30. Belonje AM, Voors AA, van der Meer P et al (2010) Endogenous erythropoietin and outcome in heart failure. Circulation 121: 245-251

31. Van der Putten K, Braam B, Jie KE et al (2008) Mechanisms of disease: erythropoietin resistance in patients with both heart and kidney failure. Nature Clin Pract Nephrol 4:47-56

32. Handelman GJ, Levin NW (2008) Iron and anemia in human biology: a review of mechanisms. Heart Fail Rev 13:393-404

33. Nemeth E (2008) Iron regulation and erythropoiesis. Current Opinion Hematol 15:169-175 